\title{
la sécurité des barrages
}

\author{
the safety of dams
}

\author{
P. LONDE*
}

Président Honoraire de la Commission internationale des grands barrages

Rev. Franç. Géotech. n 51 , pp. 41.49 (avril 1990)

\section{Résumé}

Assurer la sécurité des barrages est un souci permanent des responsables, à toutes les phases du projet, de la construction, de l'exploitation. Mais la notion de sécurité est difficile à préciser objectivement. Au surplus depuis peu les médias se font l'écho d'une large sensibilité de l'opinion publique. Dans ce rapport on aborde les différents aspects de la notion de sécurité: comment elle est ressentie par la société, ce que nous apprennent les faits statistiques, comment l'ingénieur a évolué à partir des méthodes déterministes vers une démarche probabiliste et une analyse paramétrique, enfin comment l'auscultation permet la véritable maîtrise de la sécurité.

\section{Abstract}

Ensuring the safety of dams is a major concern of all those involved, at every stage of design, construction and operation. However the safety concept is difficult to define in an objective manner. In addition, the medias recently started to reflect the high sensitivity of the public opinion. In this paper the various aspects of the safety concept are discussed: how it is accepted by the public at large, what can be learned from statistical data, how the engineers proceeded from the deterministic methods toward the probabilistic approach and the parameter study, and how instrumentation allows the real control of safety. 


\section{INTRODUCTION}

Quel que soit le type de barrage et qu'il soit en projet, en construction, en exploitation, un problème permanent est posé: celui de sa sécurité. Ce thème est sous-jacent à toutes nos démarches, plus aujourd'hui que jamais. Il stimule les développements les plus spéculatifs, il passionne les statisticiens, il motive les règlements et les cahiers des charges et, fait nouveau, il est entré dans les médias où il a une forte résonance dans la société tout entière.

Il est bien évident que dans ce contexte il n'existe pas une appréciation unique de ce qu'est en réalité la sécurité d'un barrage. Les concepts sont divers et parfois divergents. II est donc intéressant d'essayer d'approfondir d'une façon aussi objective que possible la notion de sécurité pour les barrages.

Avant d'aborder les méthodes propres à l'ingénieur, il sera bon d'analyser la manière dont le public ressent les risques liés à la présence des barrages. Nous évaluerons ensuite la situation actuelle, à partir des faits et des statistiques. Puis nous montrerons comment l'évolution des idées a conduit l'ingénieur, à partir du concept initial de coefficient de sécurité, à s'inspirer des méthodes probabilistes pour cerner de plus près la sécurité réelle. Enfin le rôle capital de l'auscultation sera souligné, car il dépasse en portée effective tous les autres progrès techniques contribuant à augmenter la sécurité des barrages.

\section{LES POSITIONS DU GRAND PUBLIC}

Le besoin de sécurité est fondamental chez l'homme. $\mathrm{Ce}$ besoin est parfaitement légitime mais il est en général exprimé d'une façon incohérente et sans base rationnelle, à partir de motivations essentiellement émotionnelles.

Depuis l'aube de l'humanité l'environnement est dangereux pour l'homme. Le progrès technique a peutêtre amélioré les choses, mais n'a pas fait disparaître les menaces. L'ours des cavernes a été remplacé par la bombe nucléaire. Le risque étant par définition le produit d'une probabilité faible par un éventuel dommage grave, il est difficile de dire si nous y avons gagné.

Quoi qu'il en soit notre environnement comporte des risques nombreux, qu'il convient, si l'on veut les dominer, d'analyser avec rigueur et objectivité. Ce n'est pas ce que fait lhomme de la rue, que nous sommes tous un peu il faut bien le dire.
Cette analyse conduit à distinguer deux grandes catégories: les risques naturels et les risques liés à l'activité humaine.

Les risques naturels: ce sont les séismes, les éruptions volcaniques, les inondations, les sécheresses prolongées, les vagues de chaleur ou de froid, les ouragans, les raz-de-marées, qui dans certains pays peuvent prendre l'ampleur d'effroyables catastrophes. Une récente statistique donne le nombre de 150000 morts par an dans le monde, en moyenne. Pendant des millénaires c'est la nature qui a été tenue pour seule responsable. Aujourd'hui on sait que, dans certains cas, la science et la technique peuvent intervenir sinon pour la prévention du moins pour la prévision, ce qui rend possible la mise en ceuvre de mesures de sauvegarde. Malheureusement ces avertissements ne sont pas toujours entendus. Nous avons tous en mémoire l'éruption du volcan Nevado de Ruiz en Colombie, en 1985, annoncée à temps pour faire évacuer la population menacée, cette prévision correcte n'ayant pas été écoutée par les autorités responsables, coûtant la vie à 22000 personnes.

Il faut dire qu'il est rare de pouvoir prédire avec certitude lampleur et la date d'un événement. La science s'exprime généralement en termes de probabilités. Or ce langage n'est pas compris des non-scientifiques.

Un bel exemple est celui du bassin du Gard en France. Les hydrologues ont pu déterminer objectivement les risques encourus par les populations riveraines des Gardons, affluents du Gard, aux crues torrentielles. Mais les périodes de paroxysme sont assez espacées pour que le souvenir s'en efface, lune après l'autre. Ce n'est qu'après les crues catastrophiques de 1958 que les pouvoirs publics ont réagi en décidant la construction d'une série de barrages de protection. Et il a fallu le désastre de Nimes lannée dernière, c'est-à-dire trente ans après, pour qu'on envisage d'achever le programme de barrages de protection.

Dans ce cas comme dans la grande généralité des cas il est étonnant de constater que c'est au coup par coup, à la suite d'événements catastrophiques, que sont prises les mesures de sauvegarde. Cela s'explique sans doute en partie par une certaine passivité de l'opinion devant les forces naturelles, associée à l'incapacité d'appréciation objective des risques.

Les catastrophes dues aux ouvrages construits par l'homme sont vécues d'une toute autre façon. Aux yeux de l'opinion ce n'est plus la nature mais nécessairement un homme qui est responsable. Si un pont ou un immeuble s'effondre, si un barrage cède, il y a fatalement un fautif ou plusieurs, soit au niveau de la conception, soit à celui de la construction, soit enfin en cours d'exploitation. Et les victimes plaident, ou l'on plaide pour elles, car il faut que justice soit faite.

Malheureusement la société n'a qu'une notion très floue de la sécurité et de ses limites. Elle est collectivement convaincue que sans faute professionnelle il n'y aurait pas de catastrophes. Cette attitude mentale est clairement traduite par la manière dont l'annonce de l'événement est faite dans la grande presse. 
La vérité objective est toute autre. Toute construction, toute activité humaine plus généralement, même si elle est conforme aux règles de l'art, contient une part de risque irréductible.

Ici intervient une distinction capitale entre le risque librement accepté par l'individu et le risque imposé par la société.

Dans la première catégorie entrent les sports dangereux ou l'usage de l'automobile. L'individu prend, de son plein gré, des risques considérables, aveuglé qu'il est par le plaisir immédiat qu'il tire de son activité. Si le varappeur qui escalade la face nord du Dru dévisse, il y aura eu faute mais sa faute. Si un chauffeur perd le contrôle de sa voiture il en sera de même. La chose se complique pour le deuxième véhicule qui sera heurté, mais ce risque est pris tous les jours pour nous tous. C'est ainsi que la route fait en France 10000 morts par an et 250000 blessés sans qu'on parle de scandale.

En revanche ceux qui vivent à l'aval d'un projet de grand barrage protestent contre le risque qui leur est imposé. C'est qu'ils n'ont pas le choix et qu'ils ne tireront pas un avantage particulier de l'ouvrage. On les comprend mais il faut les rassurer en les convainquant que le risque aujourd'hui est si petit qu'il peut être considéré comme nul. La chose n'est pas facile. La CIGB s'y emploie par la création récente d'un comité des relations publiques qui diffusera entre autres des rapports documentés sur la sécurité des barrages.

En effet les statistiques sont claires: aujourd'hui, grâce au progrès technique, les barrages sont responsables de moins de 100 morts par an pour le monde entier. Bien que la transposition soit discutable on peut estimer que pour la France seule le chiffre statistique est de un mort par an, soit 10000 fois plus faible que celui des accidents de la route.

Il est intéressant de noter que la perception du risque varie beaucoup d'un pays à l'autre. Dans notre pays il y a une tendance marquée à majorer ce risque pour des raisons émotionnelles évidentes mais exagérées. Ainsi dans la région de Fréjus on ne peut plus construire de barrage voute depuis la rupture de Malpasset en 1959. Au contraire aux Etats-Unis les cultivateurs de pommes de terre de l'Idaho demandèrent à cor et à cri la reconstruction du barrage de Teton qui s'est rompu d'une façon catastrophique en 1976. Ils en avaient besoin pour mieux vivre et étaient prêts à reprendre le risque.

\section{LES STATISTIQUES D'ACCIDENTS DE BARRAGES}

La Commission internationale des grands barrages (CIGB) s'intéresse depuis de nombreuses années à l'étude des ruptures de barrages. Les « Leçons tirées des accidents de barrages» sont régulièrement mises à jour. L'inventaire des aDétériorations des barrages et réservoirs * contient dans sa dernière édition de 1986 une liste de 107 ruptures. De ces vastes enquê- tes à l'échelle mondiale on tire des statistiques riches d'enseignements pour l'ingénieur, comme l'a fait André GOUBET dans «Les risques associés aux barrages» (La Houille Blanche, 1979).

On s'accorde à reconnaître que la plupart des ruptures résultent du concours complexe de causes multiples. Mais très souvent plusieurs mécanismes, sinon tous, ne sont pas mis en lumière avec certitude. Plus difficile encore est de déterminer la cause première, celle qui a amorcé la chaîne fatale conduisant à la catastrophe. Il est rare que des témoins occulaires crédibles puissent décrire la séquence des événements, et après l'accident la plupart des éléments faibles impliqués dans les mécanismes de rupture ont disparu. C'est pourquoi il faut être extrêmement prudent lorsqu'on cherche à tirer la leçon d'un accident. Néanmoins les statistiques, quand l'échantillon est assez grand, renseignent utilement sur les grandes tendances et les principaux pièges à éviter. Ainsi, à partir des documents de la CIGB, on peut affirmer les faits suivants.

Les barrages en remblai (terre et enrochement) étant au monde trois fois plus nombreux que les barrages en béton ils fournissent l'échantillon de ruptures le plus significatif. De l'analyse des causes on déduit que les ruptures par cisaillement, les seules qu'on sache analy. ser par le calcul, ne représentent que $15 \%$ des cas. Il est donc clair que $85 \%$ des accidents de barrages en terre résultent de faiblesses quî ne pouvaient pas être couvertes par le traditionnel coefficient de sécurité. A elles seules les actions érosives de l'eau, par insuffisance de capacité des évacuateurs ou défaut d'organes filtrants, sont à l'origine de près de $75 \%$ des accidents.

L'échantillon des barrages en béton étant beaucoup plus réduit il est plus difficile de conclure, sauf à dire que c'est la fondation qui est le plus souvent à l'origine de l'accident. On constate aussi que depuis 1960 , les barrages en béton se révèlent deux fois plus sûrs que les barrages en terre.

Une remarque s'impose ici à propos des barrages voutes. Sur les quelques 36000 grands barrages répertoriés dans le Registre Mondial de la CIGB, une seule voûte s'est rompue: Malpasset, en 1959. La voûte est statistiquement le barrage le plus sûr. S'il est vrai qu'on ne compte au total que 1500 barrages voûtes, c'est-à-dire un pourcentage de $4 \%$ seulement, ce pourcentage croît avec la hauteur. Les barrages de hauteur supérieure à $100 \mathrm{~m}$ comptent $30 \%$ de voûtes, ceux de plus de $150 \mathrm{~m}, 45 \%$ de voûtes et ceux de plus de $200 \mathrm{~m}, 55 \%$ de voûtes. Précisons qu'aucun barrage de plus de $100 \mathrm{~m}$ n'a jamais cédé, tous types confondus.

Un autre enseignement des statistiques est lamélioration de la sécurité au cours des dernières décennies. Ainsi les barrages en terre construits depuis 1960 sont 5 fois plus sûrs que ceux construits entre 1900 et 1939. La figure 1 montre cela et aussi comment les fréquences de rupture diminuent avec l'áge du barrage. On voit qu'un barrage construit au début du siècle est aujourd'hui aussi sûr statistiquement qu'un barrage récent. 


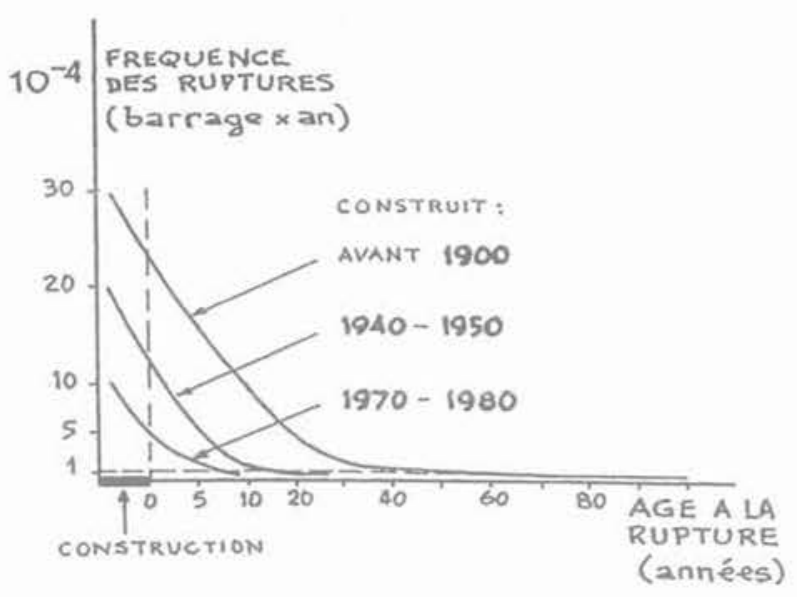

Fig. 1. - Statistique des accidents de barrages en fonction de la date de construction et de l'áge de l'ouvrage. Fig. 1. - Statistics of dam failures vs construction date and age of dam.

Actuellement le taux d'accident à l'échelle mondiale est en moyenne d'environ $5 \cdot 10^{-5}$ par barrage et par an. Il est intéressant de noter que pour les barrages construits dans la période de 1950 à 1985 les accidents ont été deux fois plus nombreux aux Etats-Unis qu'en Europe. Une explication avancée par André GOUBET serait le très fort pourcentage de barrages en terre des Etats-Unis.

Pour conclure, il convient de souligner que si les statistiques d'accidents sont précieuses pour détecter les tendances générales et guider l'ingénieur dans ses choix et ses études elles ne suffisent pas à elles seules à déterminer, dans chaque cas particulier, le niveau de sécurité d'un projet. Trop de paramètres sont présents et il faut chaque fois considérer l'ouvrage comme un exemplaire unique.

\section{LE COEFFICIENT DE SÉCURITÉ}

Nous avons vu au chapitre précédent combien les barrages en remblai étaient sensibles à d'autres types de désordres que ceux qui résultent d'une instabilité mécanique, c'est-à-dire qui sont «calculables». Mais ceci ne peut dispenser d'aborder l'analyse mécanique par des modèles mathématiques traduisant correctement les conditions d'équilibre des forces en présence. Pour les barrages en béton la démarche est capitale puisque le défaut de résistance mécanique, surtout dans la fondation, est statistiquement la cause première de désordre. Nous allons maintenant discuter de la validité de ces modèles.

Puisque la stabilité d'un barrage est intimement dépendante de la résistance de sa fondation naturelle, et qu'au surplus pour un barrage en terre son matériau constitutif est aussi naturel, le problème se pose en termes géotechniques. On ne pourra donc échapper à ce qui caractérise tout problème géotechnique:

- incertitude et dispersion dans l'espace des propriétés des sols et des roches;
- imperfection des mesures: nombre nécessairement limité et erreurs instrumentales;

- incertitude et dispersion dans le temps des actions;

- imperfection du modèle mathématique;

- grand nombre de paramètres;

- tridimensionnalité du problème.

Cet ensemble irréductible impose dans chaque cas la résolution d'un problème complexe dont les données sont incertaines et qui ne peut être traité que par des approximations elles-mêmes incertaines.

La démarche traditionnelle, et qui est encore imposée par la quasi-totalité des codes de calcul, est déterministe. Pour tenir compte des incertitudes, des dispersions et des approximations que les valeurs numériques déterminées des paramètres ne traduisent pas, on introduit le coefficient de sécurité. La marge «mesurée» par le coefficient de sécurité, entre l'état limite qu'il suffirait d'assurer s'il n'y avait aucune incertitude et l'état réel, est censée couvrir forfaitement les erreurs faites sur l'évaluation des données et par les imperfections du modèle.

La valeur numérique acceptable du coefficient de sécurité résulte de constatations empiriques. Il est admis par exemple que, d'après le comportement observé sur de nombreux ouvrages, un barrage en terre est stable avec un coefficient de sécurité, calculé par les modèles courants à l'équilibre limite, égal à 1,5. Cette valeur a même été réglementée dans certains textes qui font autorité dans le monde et qui laissent croire qu'elle assure une sécurité totale. Il n'en est rien.

D'abord pour un ensemble de données déterminé la valeur du coefficient variera avec le modèle mathématique. Or il existe une trentaine de méthodes de calcul différentes, faisant des hypothèses variées sur la distribution des contraintes internes à l'équilibre et admettant des définitions distinctes pour le coefficient de sécurité. On ne peut donc se référer à un coefficient de sécurité qu'en précisant la méthode par laquelle il a été obtenu.

Mais il y a plus grave. En effet, selon le degré d'incertitude des données (valeur de la dispersion, nombre des essais, qualité des mesures, etc.) à un même coefficient de sécurité calculé correspondent des probabilités de ruine, donc des sécurités réelles, très différentes.

La figure 2 montre dans un cas simple mais illustratif comment, pour un même coefficient de sécurité de 1,5 , la probabilité de ruine peut varier dans le rapport de 1 à 30000 , si par exemple pour une dispersion d'essais donnée on a 5 essais au lieu de 20, ou si pour 10 essais leur dispersion passe de 0,10 à 0,30 . Que pourra-t-on conclure dans le cas général et particulièrement en mécanique des roches où l'équilibre dépend d'un grand nombre de paramètres? Il est alors totalement injustifié de baser l'évaluation de la stabilité sur un nombre unique qui ne reflète pas la réalité mécanique. 


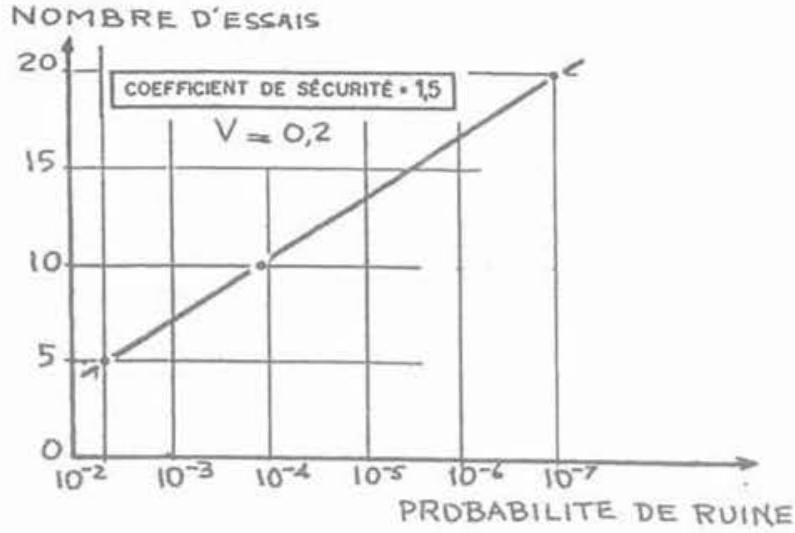

COEFFICIENT DE VARIATION $V$

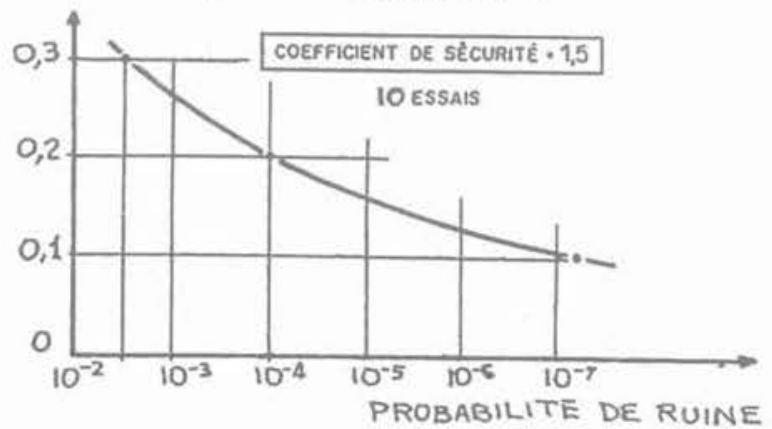

Fig. 2. - Probabilité de ruine pour un même coefficient de sécurité en fonction du nombre d'essais et de leur coefficient de variation.

Fig. 2. - Failure probability for a given safety factor, vs the tests number and their variation coefficient.

Fort heureusement les ingénieurs sont de plus en plus nombreux à prendre conscience des graves lacunes du concept de coefficient de sécurité et à reconnaître qu'il faut d'une manière ou d'une autre se rapprocher des concepts de probabilité, seuls capables de prendre en compte le caractère inéluctablement incertain des données du problème.

\section{CALCUL DES PROBABILITÉS}

Les insuffisances de la démarche déterministe, largement reconnues aujourd'hui, ont conduit de nombreux chercheurs à s'engager dans la vole des méthodes probabilistes. Leur objectif était d'évaluer quantitativement le degré de sécurité en fonction de sa véritable signification, qui est la probabilité de ruine. Au départ la notion était vague mais prometteuse car elle prenait nécessairement en compte les incertitudes de toutes sortes qui affectent les données. Au surplus, dès qu'on raisonne en probabilités, on est conduit à tenir compte objectivement des conséquences d'une éventuelle rupture. On acceptera des probabilités de ruine plus grandes si les dommages potentiels sont moindres. On peut également apprécier la variation du coût des ouvrages en fonction de leur sécurité.

Mais une difficulté majeure survient avant même d'aborder le calcul: quelles sont dans chaque cas les probabilités acceptables? On ne sait pas répondre à cette question d'une manière entièrement satisfaisante.

Il est un domaine toutefois où les ingénieurs raisonnent en termes de probabilité depuis longtemps. C'est celui des risques induits par les crues. L'observation hydrologique permet en effet d'évaluer les débits de pointe et les volumes des crues en fonction de leur période de récurrence, et l'on dimensionne les ouvrages d'évacuation pour passer la crue de la probabilité qu'on juge raisonnable en fonction des dommages potentiels que son dépassement provoqueraient. Ainsi il est d'usage pour un barrage en béton de dimensionner les évacuateurs pour la crue de 5000 ans ou de 10000 ans, alors que pour un barrage en terre, beaucoup plus vulnérable, on adopte des périodes de retour nettement plus grandes, telle celle correspondant à la notion de *crue maximale possible» (PMF en anglais) dont la période de retour, par définition, est pratiquement infinie. A linverse, pour les ouvrages provisoires on se contentera selon les risques de 10 ans, de 50 ans ou de 100 ans. Il faut dire que la crue de 5000 ans adoptée pour un ouvrage devant durer 100 ans correspond à une probabilité annuelle de $2.10^{-4} \times 10^{2}=2.10^{-2}$, égale à celle de la crue de 50 ans pour un batardeau provisoire exposé pendant un an seulement.

On a vu, par les statistiques d'accidents, que la probabilité objective de rupture est actuellement de $5 \cdot 10^{-5}$ par barrage et par an. Cette valeur très faible est à première vue du même ordre de grandeur que celle de la crue de 10000 ans. Mais un barrage destiné à être en service pendant 100 ans a une probabilité de se rompre avant 100 ans de $5.10^{-3}$ soit $1 / 200$. C'est une probabilité élevée, que nous devons nous efforcer de réduire par un progrès soutenu dans tous les domaines de la technique, y compris celui des calculs. Comme ces calculs ne couvrent qu'une partie des causes d'accident, il est évident que si l'on veut améliorer la situation actuelle, telle que révélée par les statistiques, il faut viser des probabilités de ruine ne dépassant pas $10^{-4}$ et pouvant descendre jusqu'à $10^{-6}$.

Dans cette rapide discussion se manifeste le contraste entre des probabilités d'accident qui paraissent trop élevées et qu'il faut réduire par tous les moyens en notre possession, et des probabilités si petites qu'on n'en sent pas la véritable signification. On touche là un sérieux problème des méthodes probabilistes.

Toutefois quelles que soient les difficultés de détermination des probabilités à faire entrer dans les calculs on peut être assuré qu'on cernera mieux la réalité que par un coefficient de sécurité. Par exemple, si l'on voulait diviser par dix le nombre des ruptures de remblais par glissement, nul ne pourrait dire de combien il faudrait augmenter les coefficients de sécurité traditionnels, alors que par l'analyse aléatoire on doit pouvoir trouver les moyens de le faire.

Une autre difficulté majeure est l'évaluation numérique des incertitudes. Celles-ci se traduisent par des lois de distribution des valeurs que peuvent prendre les paramètres aléatoires, lois qu'on a en général le plus grand mal à déterminer, tout particulièrement aux extrémités des courbes, c'est-à-dire pour les cas de charge ou les valeurs de résistance de faibles proba- 
bilités. Il ne faut pas oublier que la ruine résulte en général de la conjonction d'une action forte et d'une résistance faible, donc de circonstances extrêmes souvent mal définies.

\section{L'ÉTUDE PARAMÉTRIQUE}

Au terme de cette brève analyse critique des deux concepts: coefficient de sécurité, probabilité de ruine, on peut conclure que ni l'un ni l'autre ne convient pour le calcul de la stabilité des barrages. Le premier parce qu'il ne traduit nullement l'effet des incertitudes affectant les données, le second parce que, trop spéculatif, il ne peut servir pratiquement à la solution des problèmes de lingénieur. Il y a toutefois une solution: l'analyse paramétrique des équilibres limites, conduisant à la détermination des paramètres lourds et à la prise en compte correcte de l'influence de leurs incertitudes sur les conditions d'équilibre. Dans cette démarche le concept de coefficient de sécurité n'est appliqué qu'aux effets partiels et les raisonnements probabilistes ne sont utilisés que qualitativement.

Pour faciliter l'emploi des raisonnements du calcul des probabilités il est commode de considérer, au lieu du coefficient de sécurité $F$ qui est un rapport, la marge de sécurité $M$, définie comme la différence entre les résistances $\mathrm{R}$ et les actions $\mathrm{S}$. Par définition la rupture se produit si $\mathrm{M}=\mathrm{R}-\mathrm{S}$ est négatif. La probabilité $\mathrm{P}$ de cette rupture ne dépend que du coefficient de variation $V_{M}$ de la distribution de $\mathrm{M}$. On utilise souvent l'inverse du coefficient de variation, appelé coefficient de sûreté. La distribution de $M$ peut être calculée à partir des distributions des paramètres $p$ (aussi nombreux soient-ils) intervenant dans $\mathrm{R}$ et $\mathrm{S}$. Si ces paramètres sont indépendants et si les plages d'incertitude ne sont pas trop grandes on peut écrire la valeur de l'écart-type de M sous la forme:

$$
\begin{aligned}
& \sigma_{\mathrm{M}}=\left[\left(\frac{\delta \mathrm{M}}{\delta \mathrm{p}_{1}}\right)^{2} \cdot \sigma_{\mathrm{p}_{1}}^{2}+\left(\frac{\delta \mathrm{M}}{\delta \mathrm{p}_{2}}\right)^{2} \cdot \sigma_{\mathrm{p}_{2}}^{2}\right. \\
& \left.+\ldots+\left(\frac{\delta \mathrm{M}}{\delta \mathrm{p}_{\mathrm{n}}}\right)^{2} \cdot \sigma_{\mathrm{p}_{\mathrm{n}}}^{2}\right]^{\frac{1}{2}}
\end{aligned}
$$

$\mathrm{p}_{1}, \mathrm{p}_{2}, \ldots, \mathrm{p}_{\mathrm{n}}$ étant les paramètres de l'équilibre.

On peut par ailleurs calculer la valeur moyenne $\mathrm{m}_{\mathrm{M}}$ de la marge de sécurité $M$, à partir des valeurs moyennes des paramètres $p_{1}$. La connaissance de $\sigma_{M}$ et de $m_{M}$ fournit le coefficient de variation $V_{M}=$ $\sigma_{M} / m_{M}$ qui, à son tour, suffit pour chiffrer la probabilité de rupture $\mathrm{P}$. La figure 3 traduit graphiquement la relation $\mathrm{P}\left(\mathrm{V}_{\mathrm{M}}\right)$ dans le cas d'une distribution normale de $\mathrm{M}$. On peut établir les courbes correspondant à d'autres lois de distribution, mais la loi normale est correcte dans le cas général où les paramètres sont nombreux et où leurs dispersions ne sont pas excessives. On constate que la partie haute de l'échelle du coefficient de variation définit clairement les probabilités de ruine, celles-ci étant grandes, mais qu'en revanche la partie basse de cette même échelle donne une détermination très indécise. On peut en

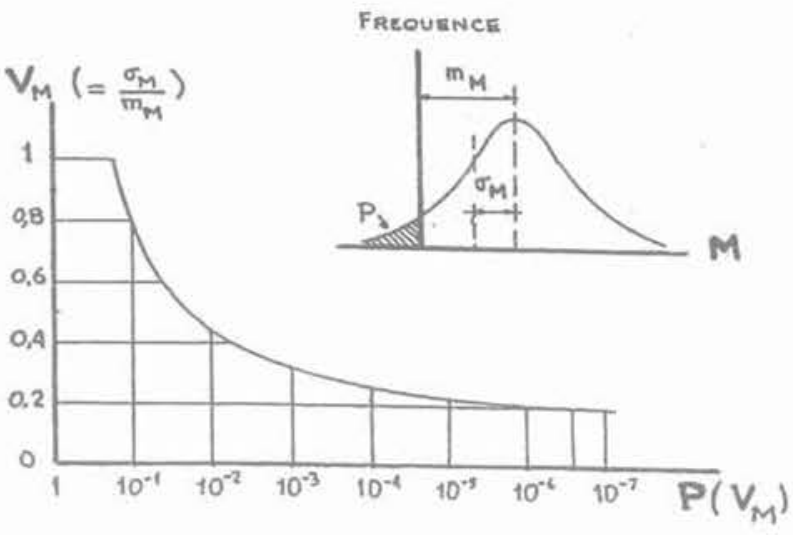

Fig. 3. - Probabilité de ruine en fonction du coefficient de variation de la marge de sécurité.

Fig. 3. - Failure probability vs the variation coefficient of the safety margin.

déduire qu'il sera impossible d'apprécier correctement les très petites probabilités. Ce résultat est conforme au sentiment, comme nous en avons discuté précédemment: les très petites probabilités sont chargées de peu de sens.

Mais le grand intérêt de cette formulation n'est pas dans l'obtention d'une valeur numérique à attribuer à la probabilité de rupture, valeur sans signification pratique. Il est dans sa structure. En effet l'équation (1), à partir de laquelle se calculerait la probabilité de ruine si on le désirait, est riche d'enseignements par sa forme même. On y voit que $\sigma_{M}$ dépend principalement des paramètres qui donnent les grandes valeurs des expressions de $\delta \mathrm{M} / \delta \mathrm{p}$ et de $\sigma_{\mathrm{p}}$. C'est-àdire que les paramètres les plus susceptibles d'influencer la sécurité sont les plus lourds $(\delta \mathrm{M} / \delta \mathrm{p}$ grand) et les plus dispersés ( $\sigma_{\mathrm{p}}$ grand). On retrouve là une règle de conduite que tout bon projeteur doit s'imposer: détecter, par une analyse même sommaire, ceux des paramètres qui pèsent le plus sur l'équilibre et traiter tout spécialement ceux qui sont mal connus, soit que leur mesure manque de précision, soit qu'elle présente une grande dispersion, soit qu'on ne puisse pas la faire d'avance.

Dans cette approche du problème on est loin du coefficient de sécurité traditionnel. Il s'écrirait $F=R / S$, les fonctions $R$ et $S$ prenant chacune une valeur déterminée, par exemple à partir des moyennes des paramètres $p_{i}$ qui ne traduit pas la réalité mécanique et ses incertitudes. Pourtant la notion de coefficient de sécurité n'a pas totalement disparu; elle se retrouve sous forme partielle, dans les dérivés $\delta \mathrm{M} / \delta \mathrm{p}_{\mathrm{i}}$, qui expriment chacune la variation de la marge de sécurité en fonction de la variation d'un seul paramètre $p_{i}$. Si donc nous ne cherchons pas à déterminer la probabilité numériquement, comme le permettraient théoriquement l'équation (1) et la figure 3 , mais si nous n'utilisons ce calcul que pour raisonner juste, nous tirons simplement parti de la forme de l'équation (1). C'est-à-dire que le projet sera conçu de façon à ce que les paramètres lourds soient tous identifiés et maîtrisés, et parmi eux tout spécialement les paramètres dispersés ou mal connus. 
L'identification des paramètres lourds peut se faire de bien des manières et pas nécessairement avec la précision du calcul complet des dérivés $\delta \mathrm{M} / \delta \mathrm{p}_{\mathrm{i}}$. Pratiquement on se sert de la variation du coefficient de sécurité traditionnel en fonction de la variation du paramètre $p_{1}$ seul. La méthode la plus sûre consiste à étudier ces variations par l'allure de graphes tracés pour les conditions de l'équilibre limite, comme on va le voir maintenant.

L'analyse paramétrique de la stabilité des massifs rocheux servant d'appui aux barrages illustre parfaitement cette méthode. Déjà ancienne et largement employée, cette méthode a été mise au point dans le domaine de la mécanique des roches, c'est-à-dire pour résoudre des problèmes caractérisés par la grande complexité des données et leurs incertitudes.

Il n'est pas nécessaire de décrire ici les détails du calcul maintes fois publiés par ailleurs. Voyons seulement cornment les résultats donnés par les graphes de la figure 4 permettent l'analyse correcte de la sécurité.

Il s'agit d'un massif rocheux dont les mécanismes de rupture sont a priori des glissements potentiels sur trois plans de discontinuité géologiques. Seuls les paramètres incertains entrent dans les graphes, $\mathrm{Ce}$ sont les trois coefficients de frottement $\varnothing_{1}, \varnothing_{2}, \varnothing_{3}$ et les trois intensités de sous-pression $\mathrm{U}_{1}, \mathrm{U}_{2}, \mathrm{U}_{3}$. Les paramètres bien connus comme les forces de masse ou la poussée du barrage ne sont pas explicités. L'équilibre limite est représenté par le quatrième sommet $r$ du rectangle construit sur les trois souspressions.

Un examen rapide de la figure 4 montre que les paramètres lourds sont $U_{1}$ et $\varnothing_{2}$. On le voit en faisant varier séparément chacun des six paramètres (noter que $\varnothing_{3}$ n'intervient pas dans l'équilibre). L'enseignement pour le projeteur est déjà précieux: il faudra agir sur la pression $\mathrm{U}_{1}$ (drainage, injection) et mesurer avec soin le frottement $\varnothing_{2}$. On pourra faire l'économie des mesures de $\varnothing_{1}$ et $\varnothing_{3}$. Il ne sera pas indispensable de drainer $\mathrm{U}_{3}$, etc. Aucune de ces indications n'aurait été obtenue à partir du calcul d'un coefficient de sécurité global, dont d'ailleurs on ne saisit pas la signification mécanique.

Ce sont évidemment les paramètres lourds qui doivent être pris avec de grands coefficients de sécurité partiels, les autres étant sans importance pour la sécurité et tolérant donc de larges erreurs d'appréciation.

Il est évident que ce type de calcul est généralisable à tout problème où entrent de nombreux paramètres. Il conduit non pas à une valeur numérique chiffrant la sécurité (tel un coefficient de sécurité ou une probabilité de ruine) mais, et c'est beaucoup plus important pour l'ingénieur, à une évaluation correcte des facteurs significatifs de l'équilibre. C'est un outil indispensable dans les cas complexes, alors que dans les problèmes simples ou répétitifs on peut utiliser les méthodes traditionnelles. Mais dans ces cas simples il est à peine besoin de calculer.

Contrairement à ce que l'on pense généralement la complexité d'un problème peut faciliter l'introduction des lois probabilistes. En effet lorsqu'un équilibre ne depend que d'un seul paramètre c'est la partie extrême de sa courbe de distribution qu'il faut évaluer puisque la ruine de la structure ne peut être due qu'à une valeur exceptionnelle de ce paramètre unique. Le problème de la détermination de la loi de probabilité est rendu difficile de ce fait, surtout si l'on s'intéresse à des valeurs aussi petites que $10^{-4}$ ou $10^{-6}$. Dans ce cas le calcul des probabilités, qui se flatte de réduire et d'interpréter correctement les effets des incertitudes, en introduit d'autres peut-être aussi graves.
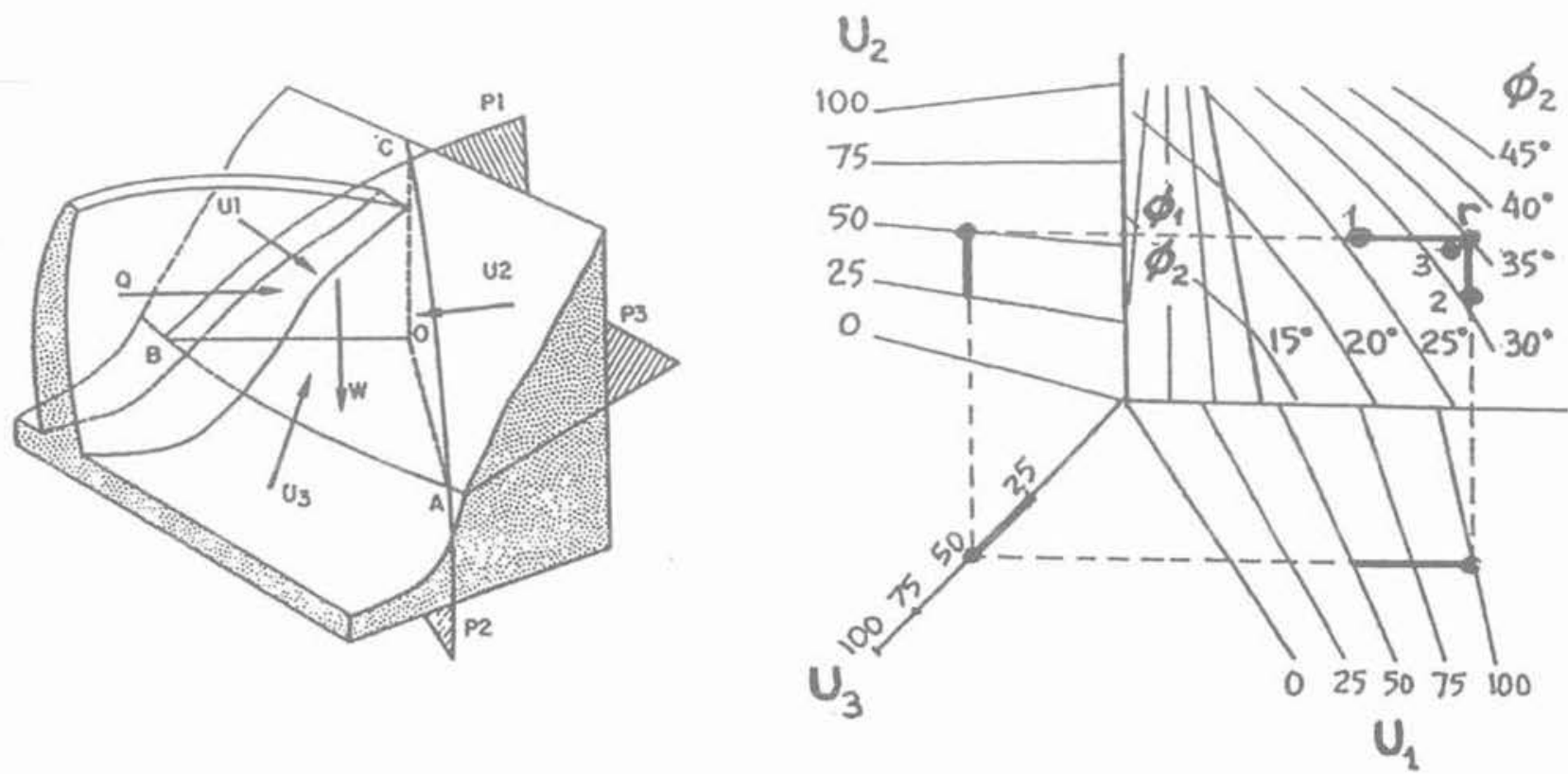

Fig. 4. - Exemple d'étude paramétrique pour un problème de mécanique des roches. Fig. 4, - Example of parameter study for a rock mechanics problem. 
Mais si, comme c'est le cas général, plusieurs paramètres significatifs interviennent dans l'équilibre l'état limite est le résultat du jeu simultané d'actions et de résistances qui, prises séparément, ne sont pas exceptionnelles. C'est leur concomitance qui l'est. On peut alors se satisfaire de lois de distribution des paramètres définies correctement dans le voisinage des valeurs moyennes sans se soucier de déterminer rigoureusement les branches extrêmes. C'est ainsi que dans le graphe proposé plus haut pour l'étude d'un appui rocheux on peut aisément introduire les distributions triangulaires ou trapézoïdales de la figure 5 , à partir d'une simple appréciation expérimentale des valeurs que peuvent prendre les frottements et les souspressions. Ici intervient l'expérience de l'ingénieur, souvent plus sure que cent essais de résistance au cisaillement ou que cent pages de calcul de pression d'écoulement. Grâce à cette simplification il est facile de faire apparaitre sur le graphe les courbes d'isoprobabilité des états d'équilibre.

L'intérêt majeur de l'analyse paramétrique est de conduire tout naturellement à l'attribution de coefficients de sécurité partiels aux paramètres qui pèsent le plus lourd dans l'équilibre et ceci en raison directe du niveau d'incertitude dont ils sont affectés. Il est évident que les paramètres parfaitement déterminés ne justifient aucune introduction de coefficient partiel.

Toutefois dans cette analyse des incertitudes du problème il reste un domaine qui échappe à une évaluation quantitative objective. Il s'agit de l'effet des approximations du modèle mécanique et à la limite de l'erreur humaine. C'est pourquoi on fera encore appel au concept de coefficient de sécurité global, mais avec une valeur beaucoup plus petite que lorsqu'il est sensé couvrir fortaitement la totalité des incertitudes du problèrne.

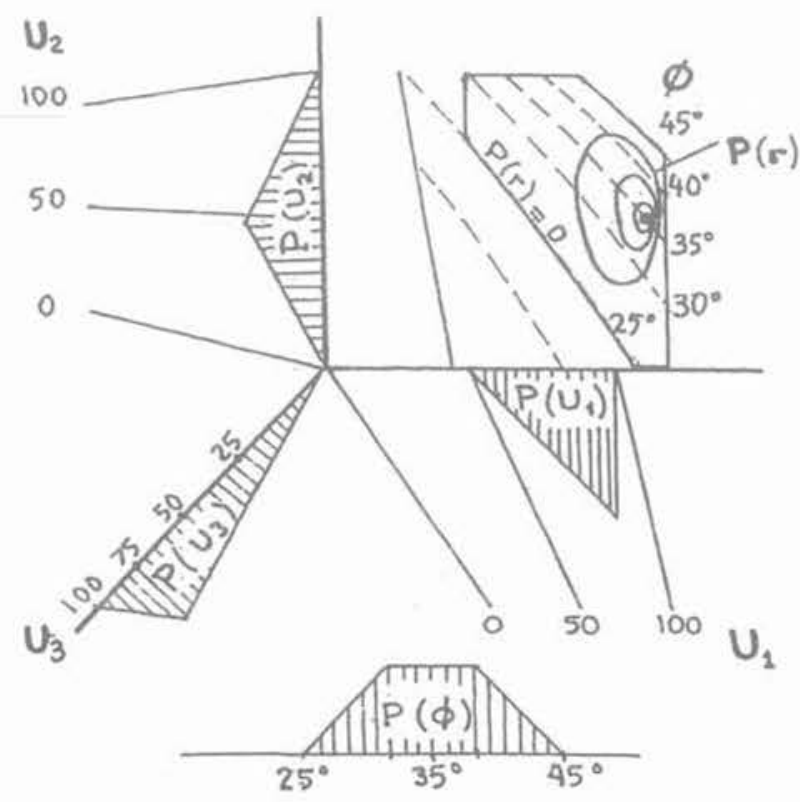

Fig. 5. - Etude paramétrique avec introduction de lois de distribution probabilistes.

Fig. 5. - Parameter study with the introduction of probabilistic density functions.

\section{L'AUSCULTATION}

On vient de voir comment les nouvelles méthodes de calcul permettaient de raffiner l'évaluation de la sécurité des barrages et de leurs fondations. Mais on a vu aussi que quoi qu'on fasse il restera toujours une part irréductible de risque de rupture, qui diminue avec les progrès techniques mais qui ne sera jamais nulle.

C'est à partir de cette constatation que s'est développée dans les récentes années une nouvelle stratégie. Le suivi du comportement mécanique des ouvrages par des instruments de mesure appropriés est considéré comme étant capital, pour indiquer à l'exploitant avant qu'il ne soit trop tard les travaux de confortement nécessaires et, dans les cas extrêmes, les mesures d'urgence assurant la protection des populations d'aval. A contrario il est frappant de constater qu'il existe une forte corrélation entre les plus graves accidents de barrage et l'absence d'instruments de mesure.

La surveillance par instruments de mesure, qu'on appelle l'auscultation, a fait des progrès considérables au cours des récentes années. Ces progrès tiennent à l'apparition de nouveaux appareils plus précis et plus fiables et surtout à la transmission des mesures à grande distance et à leur traitement informatisé. L'exploitant y a gagné en qualité de l'information et en rapidité de réaction. Pour ne citer qu'un exemple, qui est un modèle dont s'inspirent de nombreux maîtres-d'ouvrages étrangers, EDF transmet les mesures faites sur ses barrages au centre de Grenoble où elles sont traitées et immédiatement disponibles sous forme exploitable par les responsables de la sécurité.

Pour que l'auscultation soit efficace il faut qu'elle soit conçue en même temps que l'ouvrage et par les même équipes. Elle est une partie intégrée du projet. Il est également souhaitable que le projeteur participe à son interprétation, car connaissant les hypothèses mécaniques qui ont conduit au dimensionnement des ouvrages il est le mieux placé pour détecter une anomalie de comportement et en évaluer la portée.

Il est capital, pour assurer la sécurité, que les instruments mesurent des grandeurs qui soient significatives dans les mécanismes associés aux scénarios de rupture. On voit trop souvent, par exemple, installer un très grand nombre de cellules de pression interstitielle dans le noyau central des barrages en terre alors que même une surpression totale ne mettrait pas en cause la stabilité. C'est la recharge aval et particulièrement ses filtres qu'il convient d'ausculter.

De la même façon les appuis rocheux des grands barrages doivent être largement équipés de piézomètres. Ajoutons qu'aux mesures piézométriques doivent toujours être associées des mesures de débit. Leur ensemble constitue l'auscultation hydraulique qui est un des meilleurs moyens d'assurer le suivi de sécurité des barrages. 
L'interprétation des mesures et leurs signification au plan de la sécurité ne peuvent être correctement établies qu'en se référant à un modèle physique ou mathématique. Les modèles appliqués aux problèmes de stabilité mécanique font en général intervenir des contraintes. Or il est notoire qu'on ne sait pas bien mesurer les contraintes. Il y a là une sérieuse difficulté, qui a même servi d'argument pour discréditer l'auscultation dans son ensemble. La seule réponse est de faire appel à des modèles qui utilisent les paramètres les mieux mesurables. Au lieu des contraintes, toujours dispersées, il faut mesurer des déplacements ou des déformations sur de longues bases (pendules, extensomètres en forages), et se servir de modèles les prenant en compte de façon à pouvoir interpréter directement les résultats de lauscultation.

Le cas des ouvrages anciens pose un problème particulier et difficile. Or il existe dans le monde (sans la Chine) $25 \%$ de barrages âgés de plus de 40 ans et $40 \%$ âgés de plus de 25 ans. Pour beaucoup d'entre eux il faut mettre en place une auscultation ajoutée et dans un contexte délicat puisque les données du projet ont en général disparu. Un comité technique a été crée au sein de la CIGB pour l'étude de l'auscultation des barrages anciens.

On peut affirmer que l'auscultation bien conçue, associée à une surveillance par inspection visuelle systématique, augmente considérablement la sécurité réelle des barrages et le fait pour un coût négligeable en regard de l'enjeu. Elle constitue le progrès technique le plus important de ces dernières années et va certainement se traduire par une amélioration spectaculaire des résultats statistiques d'accidents, qui demeurent l'indicateur le plus significatif de notre maîtrise de la sécurité.

\section{CONCLUSION}

Pour terminer il convient de souligner le rôle capital que joue en France le Comité technique permanent des barrages, chargé du contrôle des projets, de la mise en œuvre de l'auscultation, des modalités de surveillance et des plans d'alerte. Loin de constituer une grave menace pour les riverains d'aval, comme c'était encore le cas au début du siècle, les barrages sont aujourd'hui des ouvrages très surs, en passe de devenir parmi les plus sûres des constructions humaines. A l'origine de cette amélioration spectaculaire de nombreux progrès techniques sont à citer tels que l'effort soutenu vers une meilleure compréhension du comportement des matériaux, une meilleure maitrise des méthodes de calcul et une meilleure intelligence des concepts mis en œuvre pour l'appréciation de la sécurité. Mais on a vu le rôle primordial que joue la surveillance assistée par l'ausculation, celle-ci tirant parti des progrès récents réalisés dans les appareils, la transmission des mesures et leur traitement. 